\title{
Flap selection for reconstruction of wide palatal defect after cancer surgery
}

\author{
Yun Yong Park ${ }^{1}$, \\ Hee Chang Ahn', \\ Jang Hyun Lee ${ }^{2}$, \\ Jung Woo Chang ${ }^{2}$ \\ ${ }^{1}$ Department of Plastic and \\ Reconstructive Surgery, Hanyang \\ University College of Medicine, Seoul; \\ ${ }^{2}$ Department of Plastic and \\ Reconstructive Surgery, Hanyang \\ University Guri Hospital, Hanyang \\ University College of Medicine, Guri, \\ Korea
}

\begin{abstract}
Background: The resection of head and neck cancer can result in postoperative defect. Many patients have difficulty swallowing and masticating, and some have difficulty speaking. Various types of flaps are used for palatal reconstruction, but flap selection remains controversial. Therefore, our study will suggest which flap to choose during palatal reconstruction.

Methods: Thirteen patients who underwent palatal reconstruction from 30 January, 1989 to 4 October, 2016 at our institution. Size was classified as small when the width was $<4 \mathrm{~cm}^{2}$, medium when it was $4-6 \mathrm{~cm}^{2}$, and large when it was $\geq 6 \mathrm{~cm}^{2}$. Based on speech evaluation, the subjects were divided into a normal group and an easily understood group. After surgery, we assessed whether flap selection was appropriate through the evaluation of flap success, complications, and speech evaluation.

Results: Defect size ranged from $1.5 \times 2.0 \mathrm{~cm}$ to $5.0 \times 6.0 \mathrm{~cm}$. In four cases, the defect was in the anterior third of the palate, in eight cases it was in the middle, and there was one case of whole palatal defect. There were three small defects, two medium-sized defects, and eight large defects. Latissimus dorsi free flaps were used in six of the eight large defects in the study.

Conclusion: The key to successful reconstructive surgery is appropriate selection of the flap with reference to the characteristics of the defect. Depending on the size and location of the defect, the profiles of different flaps should be matched with the recipient from the outset.
\end{abstract}

Keywords: Flaps / Microsurgery / Palate / Reconstruction

\section{INTRODUCTION}

The resection of head and neck cancer can result in postoperative defect [1]. Palatal defects are the most common, and this typically large oronasal defect causes many postoperative sequelae [2]. Many patients have difficulty swallowing and masticating, and some have difficulty speaking [3,4]. Nasal discharge can flow into the mouth through the oronasal fistula, and it has a foul odor. The condition causes pain, and compromises facial aesthetics [5]. Palatal reconstruction can solve these problems

\section{Correspondence: Hee Chang Ahn}

Department of Plastic and Reconstructive Surgery, Hanyang University College of

Medicine, 222-1 Wangsimni-ro, Seongdong-gu, Seoul 04763, Korea

E-mail: ahnhc@hanyang.ac.kr

Received August 3, 2018 / Revised September 20, 2018 / Accepted September 21, 2018
[6]. Reconstruction of the nasal lining and the oral lining is particularly important with regard to proper palatal function $[7,8]$.

Prosthetic rehabilitation has been considered the "gold standard" treatment for palatal defects [9]. However, Kornblith et al. [10] reported that prostheses were associated with poor functional outcomes. Therefore, microvascular free tissue transfer is now widely used for the reconstruction of large palatal defects. Various types of free flaps are designed for reconstruction [9, 11-13]. The most commonly used are the latissimus dorsi (LD) free flap, anterolateral thigh (ALT) free flap, and fibula free flap [14-16].

Usually, the type of flap utilized is simply based on operator preference. The current study was designed to investigate the selection of a flap that suits the characteristics of the defect, as 
opposed to flap selection based on the operator's subjective preference. It is hoped that the study will facilitate the selection of suitable free flaps depending on the size and location of the palatal defect being treated.

\section{METHODS}

In Hanyang University Medical Center, 13 patients (eight males, five females) underwent wide palatal defect reconstruction after head and neck surgery from 30 January, 1989 to 4 October, 2016. Ten patients underwent resection of palatal cancer, and three underwent palatomaxillary resection operations due to maxillary cancer. Full-layer defects of the palate ranged from $1.5 \times 2.0 \mathrm{~cm}$ to $5.0 \times 6.0 \mathrm{~cm}$. Size was classified as small when the width was $<4 \mathrm{~cm}^{2}$, medium when it was $4-6 \mathrm{~cm}^{2}$, and large when it was $\geq 6 \mathrm{~cm}^{2}$. If less than $4 \mathrm{~cm}^{2}$, the oral lining is small enough to be covered with palatal turn-over flap. So, we defined this size as "small." In case of $6 \mathrm{~cm}^{2}$, it is impossible to be covered with palatal turn over flap and two skin paddles were needed to cover the oral and nasal lining. Therefore, we used $6 \mathrm{~cm}^{2}$ as the standard for large defects.

If there was no wound remaining about 3 months postoperatively, speech evaluation was done by plastic surgeons. And we divided them into normal group, easily understood, and diffi-

Table 1. Patient demographics

\begin{tabular}{lc}
\hline Characteristics & Value $(\mathrm{n}=13)$ \\
\hline Sex & \\
Male & $8(61.5)$ \\
Female & $5(38.4)$ \\
Type of cancer & \\
Palatal cancer & $10(76.9)$ \\
Maxillary cancer & $3(23.0)$ \\
Location of defect & \\
Ant $1 / 3$ & $4(30.7)$ \\
Middle & $8(61.5)$ \\
Whole palate & $1(7.6)$ \\
Defect size $\left(\mathrm{cm}{ }^{2}\right)$ & \\
Small $(<4)$ & $3(23.0)$ \\
Medium $(\geq 4$ and $<6)$ & $2(15.4)$ \\
Large $(\geq 6)$ & $8(61.5)$ \\
\hline
\end{tabular}

Values are presented as number (\%). Ant, anterior.
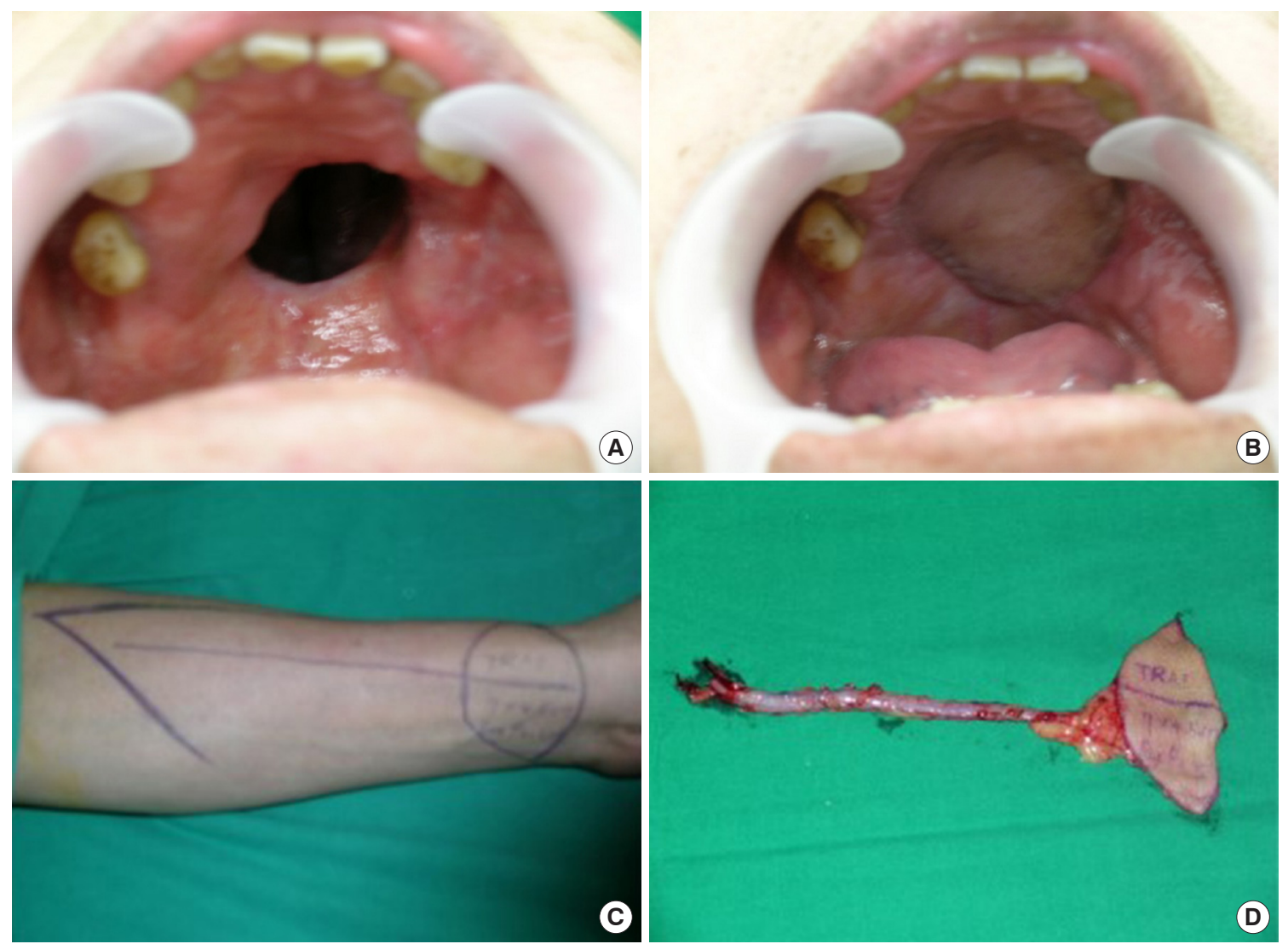

Fig. 1. Case of 48-year-old man. Palatal reconstruction with palatal turnover flap for nasal side lining and transverse radial artery forearm free flap for oral side lining. (A) Preoperative intraoral appearance. (B) Postoperative appearance in a case that was covered with a palatal turnover flap. (C) Preoperative design for transverse radial artery forearm free flap. (D) Intraoperative elevated flap. A 15-cm radial artery was elevated for the pedicle of the flap. There was one skin paddle for the nasal lining. 
cult to understood group. Patient characteristics are summarized in Table 1.

\section{Case reports}

\section{Case 1}

The first case was a 48-year-old man who underwent transverse radial forearm (TRAF) free flap for the medium sized palatal defect (Fig. 1). The pathological diagnosis was undifferentiated carcinoma. TRAFs were made with one skin paddle and used to cover the oral lining, and the nasal lining was covered with a palatal turnover flap. In oral mucosa, mucosal turnover flap is elevated to cover the defect and we sutured the flap with absorbable suture. Thereafter, the elevated TRAF free flap was insetted onto the mucosal flap. Incision was done to the mandibular angle and we were looking for the facial artery and vein. Subsequently, microscopic anastomosis was performed after preparing pedicle route through subcutaneous tunneling. The flap was then sealed using an absorbable suture. The important thing at this process was sealing tightly to make the wound waterproof. The operation time was about 5 hours and 20 minutes.

\section{Case 2}

The second case was a 54-year-old woman who underwent latissimus dorsi myocutaneous (LDmc) free flap for the large sized palatal defect (Fig. 2). After the elevation of the LDmc flap, the skin was de-epithelialized except for the portion to cover the oral lining and the nasal lining in the flap. We dissected the neck to find the recipient vessel (facial artery and vein). After finding them, the pedicle travel route was prepared with subcutaneous tunneling and microscopic anastomosis was performed. The operation time was 11 hours and 50 minutes.

\section{Case 3}

The third case was a 46-year-old man who had the whole palatal defect after maxillary cancer resection (Fig. 3). A radial osteocutaneous free flap was used when the maxilla was excised, and bony palatal reconstruction was required. We covered the flap in the same way as case 1 . In this case, harvested bone was fixed with titanium plates and screws. The operation time was 10 hours and 40 minutes.
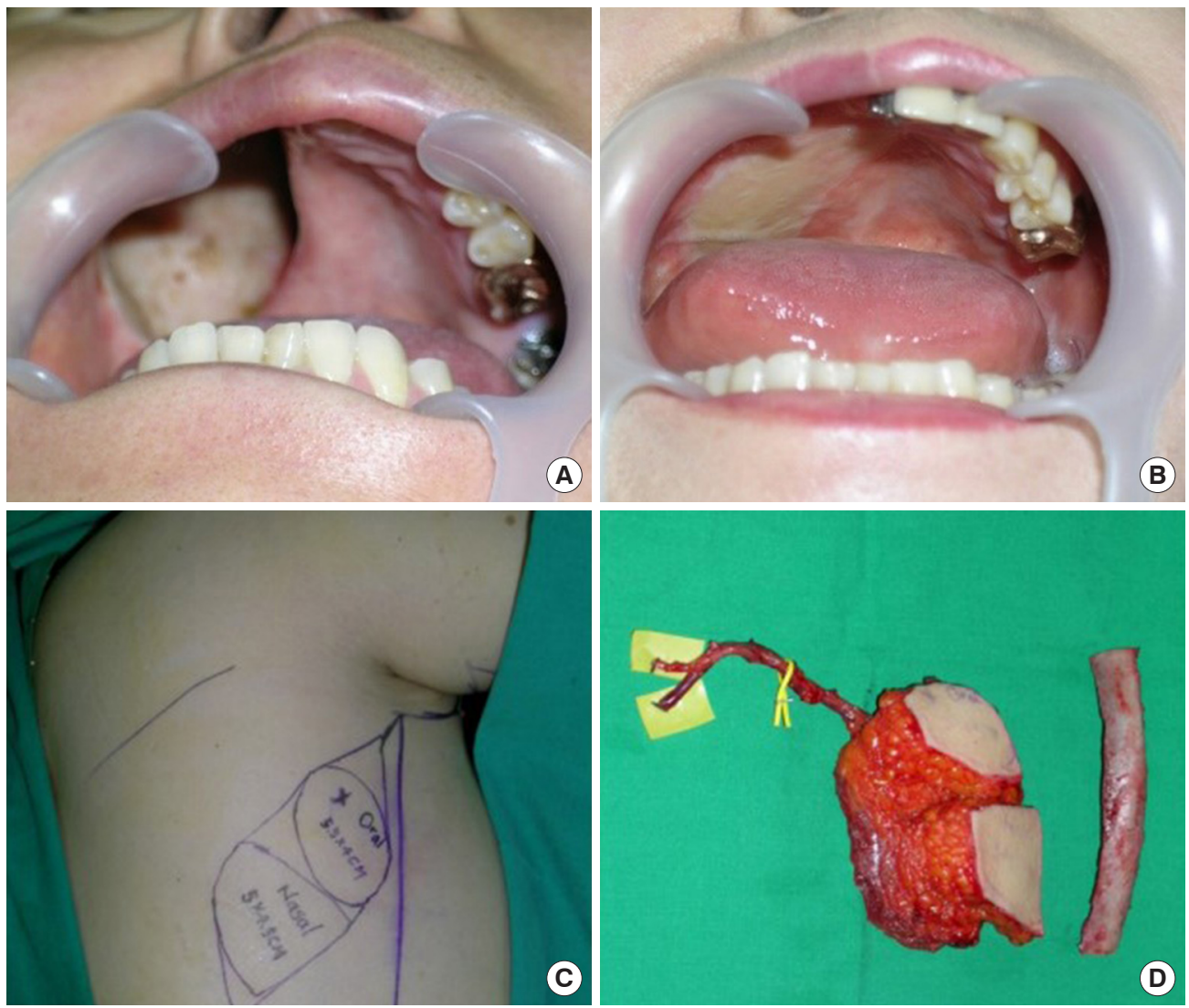

Fig. 2. Case of 54-year-old woman. Palatal reconstruction with latissimus dorsi myocutaneous free flap and rib. (A) Preoperative appearance of defects. (B) Postoperative appearance in a case that was filled with latissimus dorsi free flaps. (C) Preoperative design on the patient's flank. Two skin paddles were used; one for the oral lining and one for the nasal lining. (D) Intraoperative elevated flaps are shown, with an 8-cm thoracodorsal artery and vein for the pedicle for the flap. 


\section{RESULTS}

We performed various kinds of free flap surgery, including six LDmc free flap, five radial forearm flap, one ALT free flap, and one scapula free flap procedures (Table 2). In four cases the defect was in the anterior third of the palate, in eight cases it was in the middle, and there was one case in which the defect in- volved the whole palate and maxilla. There were three small defects, two medium-sized defects, and eight large defects. LD free flaps were used in six of the eight large defects in the study. Where bony palatal reconstruction was required, radial osteocutaneous free flaps were used. There were no cases of partial necrosis or flap failure, and there was one case of dysphagia.
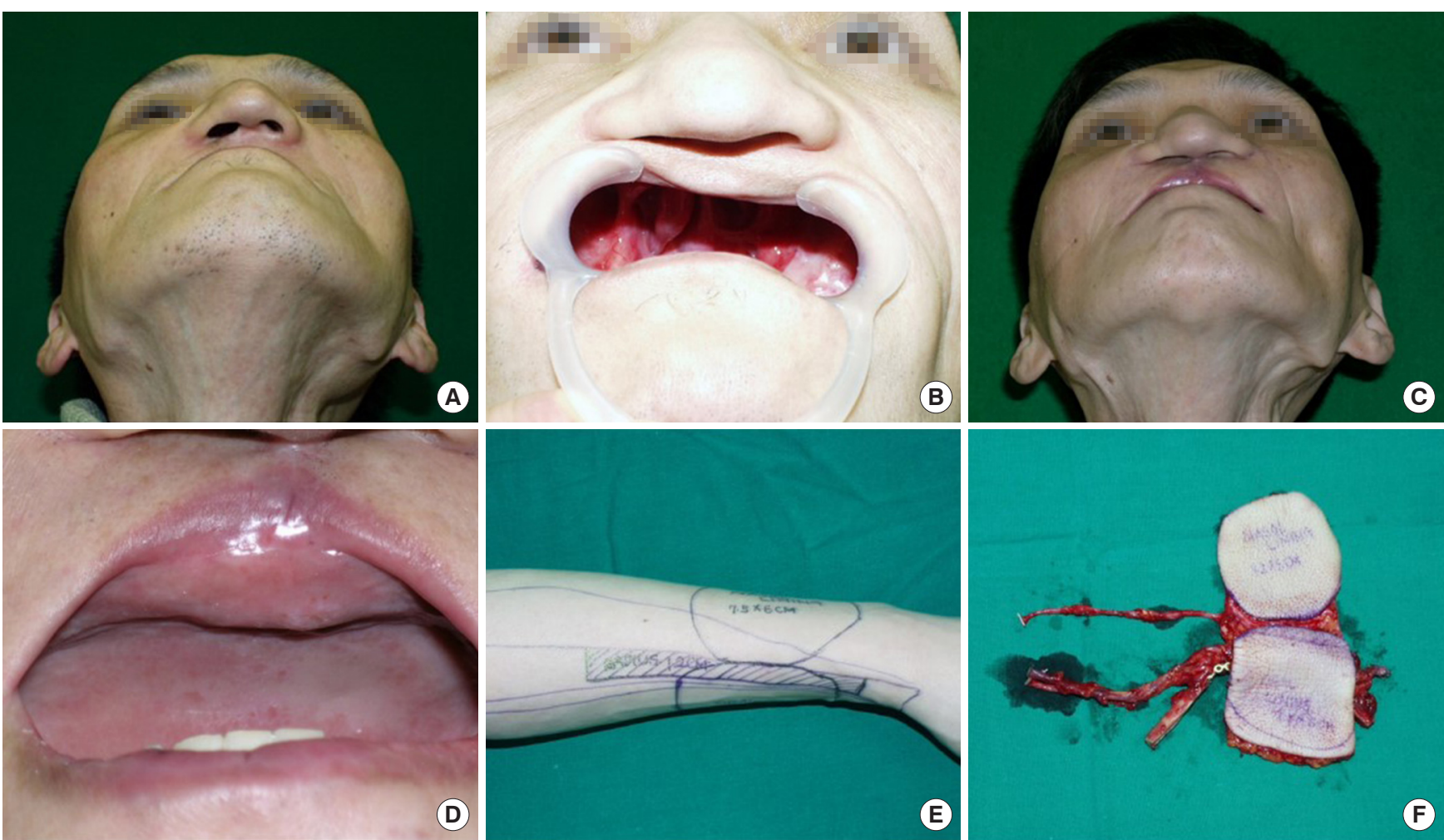

Fig. 3. Case of 46-year-old man. Palatal reconstruction with a radial osteocutaneous free flap with two skin paddles for the nasal lining and the oral lining. (A, B) Preoperative appearance. (C, D) Postoperative appearance. (E) Preoperative design of the radial osteocutaneous forearm free flap. (F) Intraoperative elevated flaps with two skin paddles for the oral lining and the nasal lining, and part of the radius, which was harvested for reconstruction of the maxilla.

Table 2. Palatal defect, patients, and methods

\begin{tabular}{|c|c|c|c|c|c|c|c|}
\hline Sex/age (yr) & Cancer & Operation & $\begin{array}{l}\text { Palatal defect } \\
\text { size }(\mathrm{cm})\end{array}$ & $\begin{array}{l}\text { Group by } \\
\text { size }\end{array}$ & $\begin{array}{l}\text { Location of defect } \\
\text { (of palate) }\end{array}$ & Speech & Complication \\
\hline $\mathrm{F} / 67$ & Palatal cancer, Rt & LDmc free flap & $3 \times 3$ & Large & Middle & Easily understood & None \\
\hline$F / 68$ & Palatal cancer, Rt & LDmc free flap and rib graft & $4 \times 3$ & Large & Middle & Easily understood & None \\
\hline $\mathrm{M} / 40$ & Palatal cancer, Rt & LDmc free flap & $3 \times 3$ & Large & Middle & Easily understood & None \\
\hline $\mathrm{M} / 46$ & Palatal cancer, Lt & LDmc free flap & $3 \times 3$ & Large & Middle & Easily understood & Dysphagia \\
\hline $\mathrm{F} / 50$ & Palatal cancer, Rt & LDmc free flap & $3 \times 3$ & Large & Middle & Easily understood & None \\
\hline$F / 54$ & Palatal cancer, Rt & LDmc free flap & $2 \times 3$ & Large & Middle & Easily understood & None \\
\hline M/68 & Palatal cancer, Rt & Palatal turn over flap+TRAF free flap & $3 \times 2$ & Large & Middle & Normal & None \\
\hline $\mathrm{M} / 48$ & Palatal cancer, Lt & Palatal turn over flap+TRAF free flap & $1.5 \times 2$ & Small & Middle & Normal & None \\
\hline $\mathrm{M} / 68$ & Palatal cancer, Rt & Palatal turn over flap+TRAF free flap & $2 \times 1.5$ & Small & Ant $1 / 3$ & Normal & None \\
\hline$M / 59$ & Palatal cancer, Lt & Palatal turn over flap+TRAF free flap & $2 \times 2$ & Medium & Ant $1 / 3$ & Easily understood & None \\
\hline $\mathrm{M} / 46$ & Maxillary cancer & Radial osteocutaneous forearm free flap & $5 \times 6$ & Large & Whole palate & Easily understood & None \\
\hline $\mathrm{F} / 68$ & Maxillary sinus cancer & ALT free flap & $2 \times 1.5$ & Small & Ant $1 / 3$ & Normal & None \\
\hline $\mathrm{M} / 59$ & Maxillary cancer, Lt & Scapula free flap & $2 \times 2$ & Medium & Ant 1/3 & Normal & None \\
\hline
\end{tabular}

F, female; M, male; Rt, right; Lt, left; LDmc, latissimus dorsi myocutaneous; TRAF, transverse radial forearm; Ant, anterior; ALT, anterolateral thigh. 


\section{DISCUSSION}

The resection of palatal cancer can result in a large defect in the palate, causing functional problems including difficulties in nose breathing, swallowing, mastication, and speech, and compromising facial aesthetics [1]. Palatal reconstruction can solve these problems [17]. One of the primary aims of palatal reconstruction is separation of the nasal and oral cavities. Without this separation, speech is altered and swallowing is hindered [7]. Accurate closure is also needed to ensure air and water tightness. It is important to preserve the soft palate and velopharynx, in order to retain velopharyngeal functions $[18,19]$. Occasionally, reconstruction of the alveolus and premaxilla are necessary. Many methods are used to reconstruct palatal defects, and it remains a controversial procedure. Obturator prostheses have been used by many surgeons; however, Kornblith et al. [10] found that prostheses were associated with poor functional outcomes. Patients with a prosthesis should maintain the hygiene of the prosthesis and the surgical site [7]. If the prosthesis is not managed properly or fixation is imperfect, leakage and oronasal regurgitation can easily occur. For this reason, reconstruction using autologous soft tissue is recommended rather than a prosthesis. Many surgeons have tried to perform palatal reconstruction using a pedicled flap near the palatal defect that preserves the vascular pedicle. However, pedicled flaps also have disadvantages when used in palatal reconstruction. Large flaps are not able to be harvested, and palatal defects cannot be completely covered. The length of the pedicle is also restricted. Therefore, microvascular free tissue transfer has been proposed as a safe method for stable palatal reconstruction $[2,5,7,20,21]$.
There is a lot of controversy about what type of free flap to choose [22]. It is generally known that free flaps including the ALT free flap, rectus abdominis muscle flap, LD free flap, and radial forearm free flap can be used to treat palatal defects [7]. In the current study, it was deemed important to determine the type of free flap used based on characteristics such as the size and location of the palatal defect. Fig. 4 shows the authors' algorithm for flap selection with regard to palatal defect.

In our study, for small and medium sized defects, coverage was performed using a turnover palatal flap for the nasal lining and a TRAF free flap for the oral lining. In the oral cavity, bulky flaps can make it difficult to speak and swallow food and drink. TRAF free flaps are not bulky, resulting in sufficient space in the oral cavity [7]. Notably, if radiation therapy has been applied after palatal cancer surgery, the tissue tends to be unstable due to radiological damage, and the condition of blood vessels is not conducive to good anastomosis. In such cases, a long pedicle is used in conjunction with a radial forearm flap, and anastomosis is performed in a healthy tissue area that was not subjected to radiation therapy.

For large defects, coverage was performed using LD free flaps. In these cases, a large flap could be elevated and divided into two skin paddles to cover both the nasal lining and the oral lining. Palatal turnover flaps that fit the defect sizes were not able to be harvested in cases of large defects, so we harvested large flaps to reconstruct both the oral lining and the nasal lining. If there was a bony defect such as bimaxillary defect as in case 4, it was difficult to correct the depression deformity by soft tissue transfer alone. Therefore, a radial osteocutaneous forearm free flap, an LD free flap with a rib graft, or a fibula free flap can be

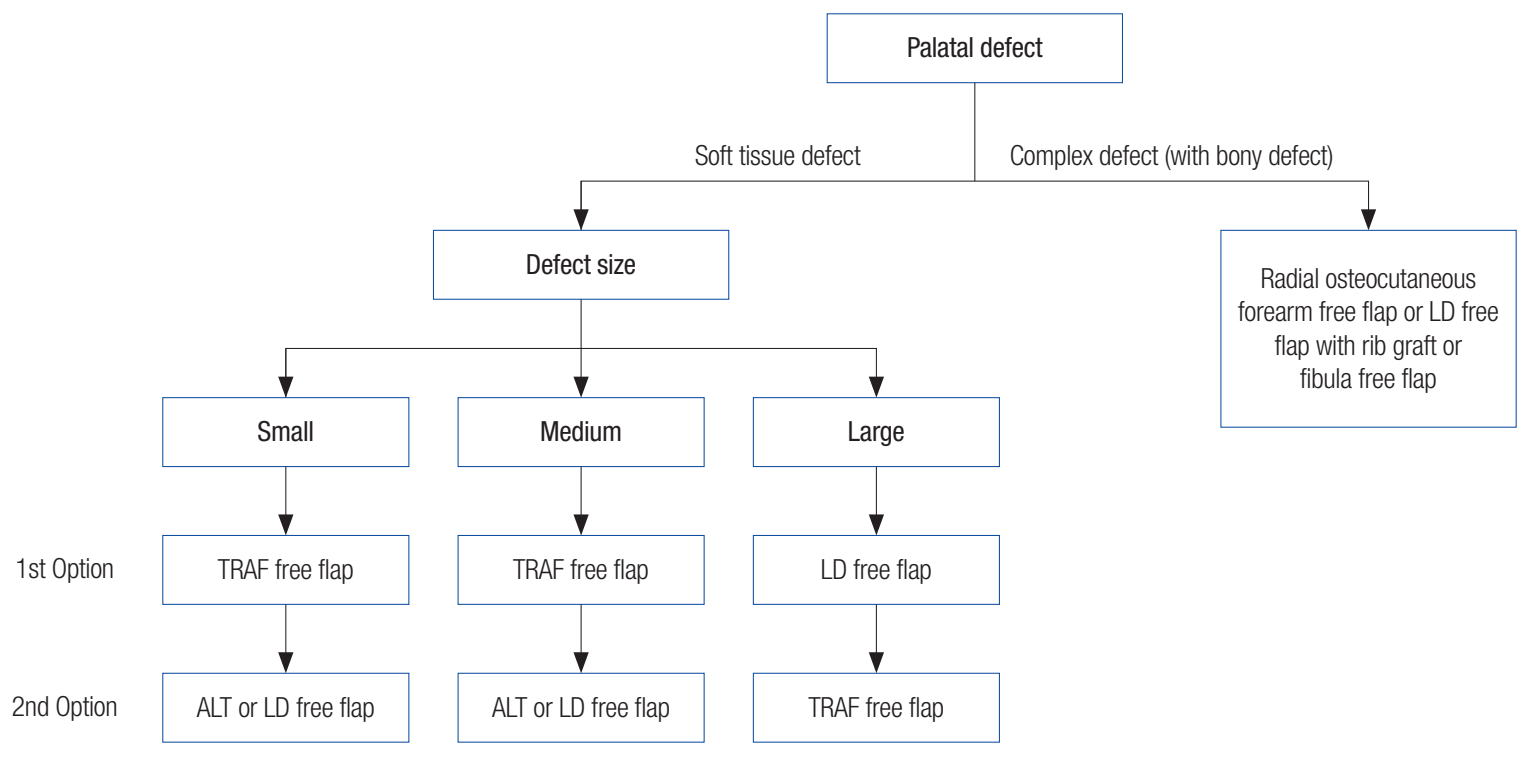

Fig. 4. Algorithms for flap selection. LD, latissimus dorsi; TRAF, transverse radial forearm; ALT, anterolateral thigh. 
performed. In one case, we attempted to perform palatal turn over flap and TRAF free flap for the patient who had small defect, but she refused and underwent ALT free flap according to the second choice in our protocol. In another case, patient who had medium sized defect refused our first choice (palatal turn over flap and TRAF free flap). So, we suggested several options for the reconstruction. As a result, he chose scapula free flap.

In our study, we tried to derive the results according to the location of the palatal defect, but did not make a meaningful result for the location. There were no complications such as flap failure or partial necrosis of the flaps in the current study. One patient had dysphagia, which was evidently caused by bulky flaps. Specifically, the patient complained of dysphagia because he could not adjust to the bulky flap after surgery.

The current study had some limitations. The number of cases was small, partly because the prevalence of cancer of the oral cavity is only approximately 10 persons per 100,000 per year [23], and palatal cancer only constitutes a proportion of those cases. Therefore, the number of defects due to palatal cancer is low, and only a few patients undergo major operations for reconstruction. We did not observe various complications due to the low number of cases. Another limitation was the lack of objective speech evaluation after surgery. In general, speech evaluation is performed via an automatic speech recognition system, but in the present study this form of objective evaluation was not performed.

This study is valuable with regard to palatal reconstruction. Previous studies have mainly focused on the introduction of flaps in palatal defects, and due to low numbers of cases, flap procedures were performed in accordance with the operator's subjective preference. No previous study investigating flap selection based on defect characteristics such as size and position has been reported. In addition, using the palatal reconstruction procedures described in the current report it is possible to preserve the function of the palate based on an understanding of the anatomy. Because of the characteristics of the palate, as well as the reconstruction filling the required volume, it is also important to reconstruct the oral and nasal lining to preserve function by separating the oral cavity and the nasal cavity. Palatal function was preserved via our reconstruction procedures.

Herein, we described the broad spectrum of free flaps available for palatal reconstruction after head and neck cancer surgery. The key to successful reconstructive surgery in this context is appropriate selection of the flap based on the characteristics of the defect. Depending on the size and location of the defect, profiles of different flaps should be matched with the recipient from the outset.

\section{NOTES}

\section{Conflict of interest}

No potential conflict of interest relevant to this article was reported.

\section{Ethical approval}

The study was approved by the Institutional Review Board of Hanyang University Medical Center (IRB No. 2018- 06-033) and performed in accordance with the principles of the Declaration of Helsinki. Written informed consent was obtained.

\section{Patient consent}

The patients provided written informed consent for the publication and the use of their images.

\section{ORCID}

Yun Yong Park https://orcid.org/0000-0003-3594-5891

Hee Chang Ahn https://orcid.org/0000-0002-6810-5752

Jang Hyun Lee https://orcid.org/0000-0002-0552-4554

Jung Woo Chang https://orcid.org/0000-0002-7937-9679

\section{REFERENCES}

1. Ozkan O, Ozkan O, Coskunfirat OK, Hadimioglu N. Reconstruction of large palatal defects using the free anterolateral thigh flap. Ann Plast Surg 2011;66:618-22.

2. Futran ND, Mendez E. Developments in reconstruction of midface and maxilla. Lancet Oncol 2006;7:249-58.

3. Germain MA, Hartl DM, Marandas P, Julieron M, Demers G. Free flap reconstruction in the treatment of tumors involving the hard palate. Eur J Surg Oncol 2006;32:335-9.

4. Rieger JM, Zalmanowitz JG, Li SY, Tang JL, Williams D, Harris J, et al. Speech outcomes after soft palate reconstruction with the soft palate insufficiency repair procedure. Head Neck 2008; 30:1439-44.

5. Lenox ND, Kim DD. Maxillary reconstruction. Oral Maxillofac Surg Clin North Am 2013;25:215-22.

6. Moore BA, Magdy E, Netterville JL, Burkey BB. Palatal reconstruction with the palatal island flap. Laryngoscope 2003;113: 946-51.

7. Futran ND, Haller JR. Considerations for free-flap reconstruction of the hard palate. Arch Otolaryngol Head Neck Surg 1999;125:665-9.

8. Gupta V, Cohan DM, Arshad H, Kuriakose MA, Hicks WL Jr. Palatal reconstruction. Curr Opin Otolaryngol Head Neck Surg 2012;20:225-30.

9. Bernhart BJ, Huryn JM, Disa J, Shah JP, Zlotolow IM. Hard 
palate resection, microvascular reconstruction, and prosthetic restoration: a 14-year retrospective analysis. Head Neck 2003; 25:671-80.

10. Kornblith AB, Zlotolow IM, Gooen J, Huryn JM, Lerner T, Strong EW, et al. Quality of life of maxillectomy patients using an obturator prosthesis. Head Neck 1996;18:323-34.

11. Muzaffar AR, Adams WP Jr, Hartog JM, Rohrich RJ, Byrd HS. Maxillary reconstruction: functional and aesthetic considerations. Plast Reconstr Surg 1999;104:2172-83.

12. Elsherbiny M, Mebed A, Mebed H. Microvascular radial forearm fasciocutaneous free flap for palatomaxillary reconstruction following malignant tumor resection. J Egypt Natl Canc Inst 2008;20:90-7.

13. Seikaly H, Rieger J, Wolfaardt J, Moysa G, Harris J, Jha N. Functional outcomes after primary oropharyngeal cancer resection and reconstruction with the radial forearm free flap. Laryngoscope 2003;113:897-904.

14. Genden EM, Wallace D, Buchbinder D, Okay D, Urken ML. Iliac crest internal oblique osteomusculocutaneous free flap reconstruction of the postablative palatomaxillary defect. Arch Otolaryngol Head Neck Surg 2001;127:854-61.

15. Granick MS, Ramasastry SS, Newton ED, Solomon MP, Hanna DC, Kaltman S. Reconstruction of complex maxillectomy defects with the scapular-free flap. Head Neck 1990;12:377-85.

16. Cordeiro PG, Santamaria E, Kraus DH, Strong EW, Shah JP.
Reconstruction of total maxillectomy defects with preservation of the orbital contents. Plast Reconstr Surg 1998;102:1874-84.

17. Bakamjian V. A technique for primary reconstruction of the palate after radical maxillectomy for cancer. Plast Reconstr Surg 1963;31:103-17.

18. Sinha UK, Young P, Hurvitz K, Crockett DM. Functional outcomes following palatal reconstruction with a folded radial forearm free flap. Ear Nose Throat J 2004;83:45-8.

19. McCombe D, Lyons B, Winkler R, Morrison W. Speech and swallowing following radial forearm flap reconstruction of major soft palate defects. Br J Plast Surg 2005;58:306-11.

20. Genden EM, Okay D, Stepp MT, Rezaee RP, Mojica JS, Buchbinder D, et al. Comparison of functional and quality-of-life outcomes in patients with and without palatomaxillary reconstruction: a preliminary report. Arch Otolaryngol Head Neck Surg 2003;129:775-80.

21. Shestak KC, Schusterman MA, Jones NF, Johnson JT. Immediate microvascular reconstruction of combined palatal and midfacial defects using soft tissue only. Microsurgery 1988;9: 128-31.

22. Davison SP, Sherris DA, Meland NB. An algorithm for maxillectomy defect reconstruction. Laryngoscope 1998;108:215-9.

23. Neville BW, Day TA. Oral cancer and precancerous lesions. CA Cancer J Clin 2002;52:195-215. 